\title{
Kinetics of Changes in Acceleration Photoplethysmogram Indices Elicited by Pericardium 6 Acupoint
}

\author{
José Federico Rivas-Vilchis ${ }^{1 *}$, Marcial Mejía-González² \& Felipe Juárez Lozano² \\ ${ }^{\text {I}}$ Posgrado en Acupuntura y Fitoterapia, Unidad Iztapalapa, Universidad Autónoma Metropolitana, Ciudad de México, México. \\ ${ }^{2}$ Escuela de Medicina Natural Integrativa, Universidad Evangélica Nicaragüense Martin Luther King (UENIC MLKJr), Campus \\ Mazorca Calpulli A.C., Ciudad de México, México. ${ }^{1 *}$ Corresponding authoremail: jfrv@xanum.uam.mx
}

DOI: http://doi.org/10.38177/ajast.2021.5204

Copyright: @2021 José Federico Rivas-Vilchis et al. This is an open access article distributed under the terms of the Creative Commons Attribution License, which permits unrestricted use, distribution, and reproduction in any medium, provided the original author and source are credited.

\section{ABSTRACT}

Objective: To observe the kinetics of acupuncture effect at the pericardium 6 acupoint on the acceleration photoplethysmogram (APG) indices in healthy people.

Subjects and methods: Photoplethysmography pulse waveforms were recorded non-invasively for 20 min from the right index finger in eight normal healthy human subjects. The AGP-b/a, AGP-d/a, and the ADP-AI indices based on the height of the photoplethysmogram wave components were calculated. Manual acupuncture of Pericardium 6 acupoint during 5 min contralateral to photoplethysmograph registration was applied. The APG indices values at basal versus acupuncture stimulation period and posacupuncture were compared using the t-student test.

Results: APG-b/a and APG-AI indices increased during acupuncture stimulation and decreased after needle withdrawal; both changes were no significant. Otherwise, APG-d/a steadily decreased during acupuncture and posacupunture, and comparing basal versus 20 min values, there was a significant difference $(p=0.031)$

Conclusion: The results show that manual acupuncture in PC6 acupoint acutely modifies APG indices and the FFT-smoothed spectrum of APC indices can be taken as a kinetic modeling to study the time-sequence of the effects of acupuncture on cardiovascular variables.

Keywords: Acceleration photoplethysmogram, Arterial stiffness, Plethysmogram, Pericardium 6 acupoint, Pulse wave.

\section{Introduction}

Photoplethysmography is an optical non-invasive technique for monitoring blood perfusion in the skin, changes in the intensity of the reflected light are related to blood flow, blood volume, and blood vessel wall movement in the underlying tissue [1]. Photoplethysmographic (PPG) waveform analysis has been used for estimating peripheral arterial stiffness [2]. The second derivative of photoplethysmogram (SDPTG) or second derivative of digital volume pulse (SDDVP) has been called acceleration plethysmogram (APG) [3].

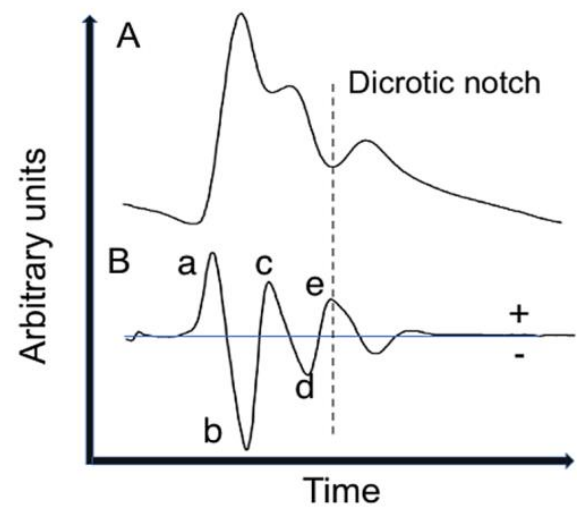

Fig.1. The five APG waves

The APG consists of five waves, including APG-a wave (early systolic positive wave), APG-b wave (early systolic negative wave), APG-c wave (late systolic re-increasing wave), APG-d wave (late systolic re-decreasing wave), 


\section{A TAST}

and APG-e wave (early diastolic positive wave) [4], Figure 1. Normalized amplitudes b/a have a positive and c/a, $\mathrm{d} / \mathrm{a}$, and e/a have a negative correlation to the age; and APG-b/APG-a ratio indicates raised arterial rigidity, and the APG-b/APG-a ratio rose with age [4]. The APG-b/a index is associated with the peripheral arteries distensibility, and it has been proposed as a valuable non-invasive index of atherosclerosis and changes in arterial compliance [5]. The APG-d/APG-a ratio demonstrates the intensity of reflecting pulse waves from peripheral arteries [6]. The following formula can calculate APG aging index: [(APG-b)-(APG-c)-(APG-d)-(APG-e)]/(APG-a) and is proposed to signify the distensibility of large arteries [4].

Figure 1:- Representative trace of the digital volume pulse and the second derivative of the photoplethysmogram or acceleration photoplethysmogram (APG). The APG wave comprises five components from: positive waves a, c, and $e$; negative $b$ and $d$. APG-b/a index is calculated with the quotient of $b$ and $a$; the APG-d/a index is calculated with the quotient of the relative values of the waves $d$ and $a$. The aging index or APG-AI was defined as the quotient of the algebraic sum of (b-c-d-e) between a.

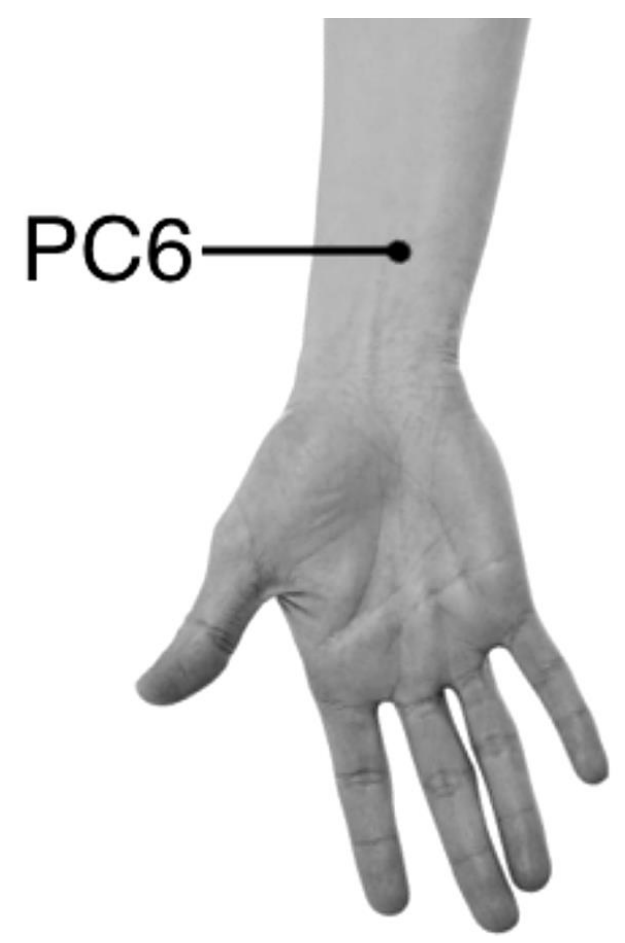

Fig.2. Pericardium 6 (PC6) acupoint.

Clinical and experimental observations have suggested that acupuncture may have therapeutic effects on various types of hypertension, coronary heart disease, dysrhythmias, and myocardial infarction [7, 8]. Besides, the PC6 acupoint (PC6) is one of the most commonly used acupoints, is indicated for treating cardiovascular-related disorders (Figure 2), and is one of the primary acupoints used to treat cardiovascular diseases [9, 10]. PC6 is also an acupoint commonly used to study the effects and mechanisms of acupuncture in several different animal models of cardiovascular disease [11, 12]. Although several studies report the effects of PC6 [13 - 15], its mechanisms and are not fully understood. Furthermore, to our knowledge, the kinetics of the cardiovascular effect point PC6 has not been described. The primary objective of this study is to evaluate the kinetics of P6 manual acupuncture on the APG indices in healthy subjects. 


\section{Methods}

Study design. This study is designed as an experimental non-randomized, single-center trial to check the kinetics of manual acupuncture in right PC6 on the APG indices in healthy subjects.

Subjects. The study group comprised 8 ( 4 female) healthy subjects with a mean ( \pm SD) age of $44.6 \pm 7.2$ years. All subjects were normotensive (office blood pressure $<140 / 90 \mathrm{mmHg}$ ), none had total serum cholesterol $>200 \mathrm{mg} / \mathrm{dl}$, neither glucose $>100 \mathrm{mg} / \mathrm{dl}$ at the moment of the study. None had cardiac or renal diseases or were taking any medications. The exclusion criteria were the presence of cardiovascular or renal diseases.

Ethical aspects. The Ethics Committee of Health and Biological Sciences Division, Metropolitan Autonomous University at Iztapalapa (2007.6.01.03) approved the trial. All participants granted a signed informed consent.

Instrumentation and recordings. The second derivative of the PPG wave contour was obtained using the Origin, Scientific Graphing, and Analysis Software, v. 7.5 (Microcal Software, Inc., Northampton, MA) to specify inflection points. Typically, the $\mathrm{d}^{2} \mathrm{PPG} / \mathrm{dt}^{2}$ waveform comprises five distinct waves: a to e. Each wave was measured from the baseline, the values above the baseline being positive and those under it negative, as previously described [4].

PPG register. The photoplethysmogram detects absorption changes of light by hemoglobin, which reflects changes in blood flow volume. A photoplethysmography transducer (TSD 200; BIOPAC Systems, Goleta, CA, USA) that transmits infrared light at $860 \pm 90 \mathrm{~nm}$ was used to obtain the PPG. The transducer was placed on the index finger of the right hand of each experimental subject. The frequency response of the photoplethysmograph was flat at $10 \mathrm{~Hz}$. The digital output of the photoplethysmograph was recorded through a 12-bit analog-to-digital converter with a sampling rate of 200 points per second (MP100; BIOPAC Systems, Goleta, CA, USA). The analysis platform provided by Acknowledge software version 3.8.1 (MP100; BIOPAC Systems, Goleta, CA, USA) was used.

APG indices. The second derivative of the PPG wave was obtained using Origin software, Scientific Graphing and Analysis Software version 7.5 (Microcal Software, Inc., Northampton, MA, USA). The $\mathrm{d}^{2} \mathrm{PPG} / \mathrm{dt}^{2}$ or APG wave typically comprises five distinct waves: a to e. The APG indices are calculated using the relative height of the positive or negative inflections. The values of b-wave and d-wave are normalized to a-wave, then the ratios b/a and $\mathrm{d} / \mathrm{a}$ are obtained. The aging index of the second derivative (APG-AI) is defined as the quotient of the algebraic sum (b-c-d-e)-waves normalized with the a-wave value [4].

The data of each subject corresponding to each min were plotted, and the interpolated data for the eight subjects were averaged with the software Plot2 (Michael Wesemann, Berlin, Germany, 2019), and a kinetic curve of the acupuncture effect was obtained. Subsequently, the curves corresponding to each variable were gathered in a single graph.

Intervention. PC6 was chosen because of its well-known cardiovascular effects. We used 5-cm-long, 0.22-mm-wide disposable stainless steel needles (SHARP, Suzhou, Beijing, China) with no additional electrical or laser stimulation. The diagnostic workup included the recording of medical history and symptoms. An acupuncture 
needle was inserted unilaterally into PC6 (Figure 2) at $1.5 \mathrm{~cm}$ deep in the right arm and was manually stimulated until acupuncture characteristic sensation (DeQi) was obtained. The needle was maintained for $5 \mathrm{~min}$ (1-6 min) and removed after that. All subjects were given the same amount of attention and received the same acupuncture treatment. The trial was blinded for clinical investigator calculating APG indices. Acupuncture treatment was given by a physician trained in both orthodox Western medicine and traditional Chinese medicine.

Study protocol. All subjects were studied fasting, having abstained from caffeine, alcohol, or smoking in the previous $12 \mathrm{~h}$. The measurements were performed with each subject in the supine position. All recordings were made in the morning in a laboratory with a temperature of $24 \pm 1{ }^{\circ} \mathrm{C}$. All subjects rest for at least 30 min before recordings commenced. A photoplethysmograph TSD200 (BIOPAC Systems, Inc., Goleta, CA, United States) transmitting infrared light at $860 \mathrm{~nm} \pm 90 \mathrm{~nm}$ placed on the left hand index finger was used to obtain the PPG. The frequency response of the photoplethysmograph was flat to $10 \mathrm{~Hz}$.

The digital output from the photoplethysmograph was recorded through a 12-bit analog-to-digital converter with a sampling frequency of 200 points per second BIOPAC Systems, MP150 (BIOPAC Systems, Inc., Goleta, CA, United States) using AcqKnowledge v. 4.1 software (BIOPAC Systems, Inc., Goleta, CA, United States). Of each subject, a PPG continuous registration 20 min long was obtained. After a 1 min basal record, PC6 was stimulated in the right arm manually until obtaining the DeQi reaction, contralateral to the PPG registry side. PC6 right was stimulated by manual needling for $5 \mathrm{~min}$ (1-5 min). Continuous and high-quality APG indices were calculated from beat-to-beat PPG and calculated for every subject in the basal (pre-acupuncture) and posacupuncture periods (minutes 8, 10, 12, 14, 16, 18, and 20). The APG indices for each group were averaged and then tested for statistically significant differences between the two groups. Given the wide variation of the APG indices to obtain a reliable version of the peaks and their intensities, a smoothed function through the Fast Fourier Transform of the signal for each data set of APG indices was obtained.

Outcomes. The APG-b/a, APG-d/a, and APG-AI indices were calculated in the basal period, each minute during the acupuncture period, and minutes $8,10,12,14,16,18$, and 20 of the posacupuncture period.

Data analysis. Based on absolute values for the height of waves a, b, c, and d of the raw PPG, we comprehensively calculated and analyzed the following variables: APG-b/a (i.e., the ratio of the height of the $b$ wave to that of the b); and analogously, APG-d/a; and APG- (b-c-d-e)/a (the so-called aging index or AGI) (4). Representative traces of the photoplethysmogram and its second derivative are shown in figure 1 .

Data interpolation. The records of the subjects of each group were plotted in the Plot2 software (Michael Wesemann, Berlin, Germany, 2019). The interpolated data of the eight subjects of the experimental group were averaged, and kinetic curves of the behaviour of the APG indices were obtained.

Baseline values were compared with those corresponding to 1, 2, 3, 4, 5 min of the acupuncture stimulation period, and 2, 4, 6, 8, 10, 12, 14 min after needle withdrawal.

Smoothing of pooled data. The interpolated data of each APG index was smoothed through fast Fourier transform in the Plot2 software (Michael Wesemann, Berlin, Germany, 2019). 
Statistical Analysis. Data are reported as means \pm standard deviation (SD). The Student t-test was used for comparison of normally distributed continuous variables. The chosen level of statistical significance was $\mathrm{p}<0.05$. All statistical analyses were performed using the statistical package for social sciences (SPSS, version 22.0, SPSS Inc., Chicago, IL, USA).

\section{Results}

APG-b/a index. The changes in the APG-b/a index and the smoothed template using the fast Fourier transformation during and after manual acupuncture in PC6 are depicted in Figure 3. The acupuncture treatment elicited an APG-b/a increase, and after needle withdrawal, a diminution; both changes were no significant. Imanaga et al. [5] provided direct evidence that the magnitude of b/a of the APG is related to the distensibility of the peripheral arteries and suggested that the extent of APG-b/a is a useful non-invasive index of atherosclerosis and arterial stiffness and hypertension. Besides, these changes could be related to modifications of mean arterial pressure [16].

Besides, the smoothing of the APG-b/a index showed a biphasic behaviour with an increase in the acupuncture period and a secondary decrease response. Smoothing of noisy data using Fourier Transform has a long history: from empirical algorithms of Lanczos [17] to using polynomial filters [18]. Nowadays, the position of the peaks and their intensities measured in the smoothed spectra have high reliability.

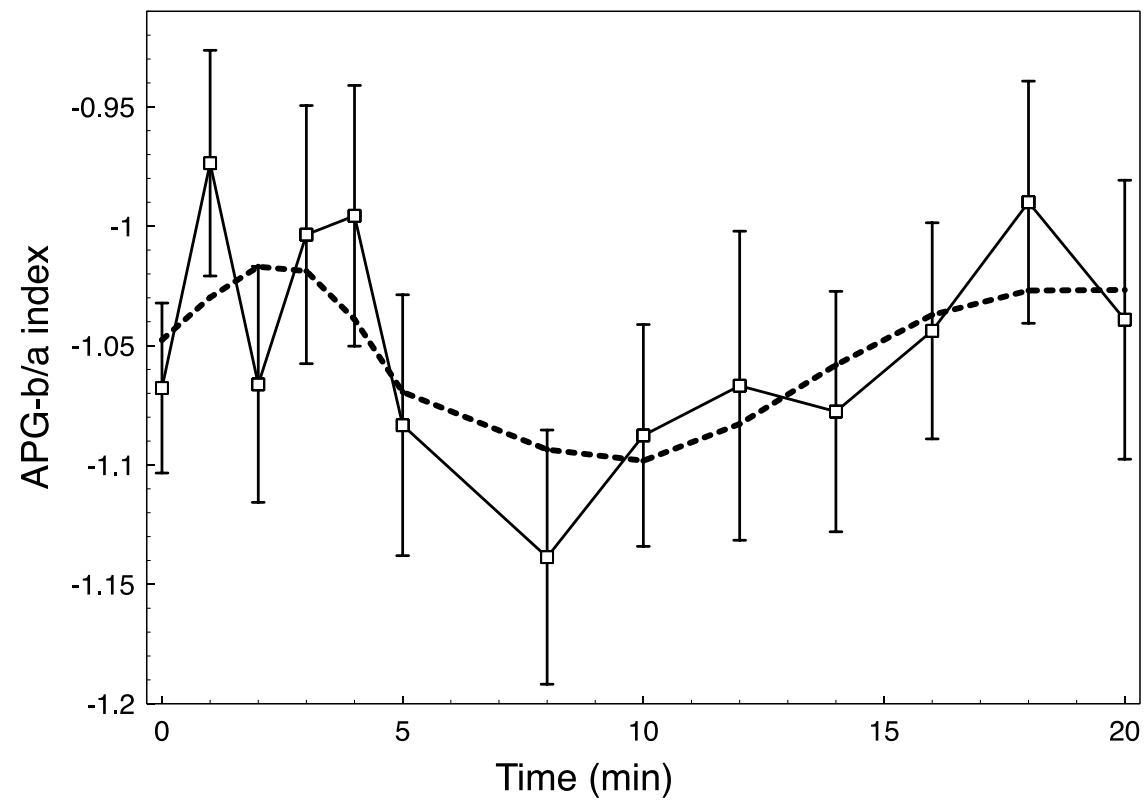

Fig.3. The second derivative of photoplethysmogram or acceleration photoplethysmogram b/a (APG-b/a) index with standard error. Smoothed template using the fast Fourier transformation (dotted line).

APG-d/a index. The changes in the SDPP- $d / a$ index and the smoothed template using the fast Fourier transformation during and after manual acupuncture in PC6 are depicted in Figure 4. The acupuncture treatment elicited an APG-b/a steady diminution with a further decrease after needle withdrawal. Comparing basal versus value at the end of the study, there was a significant decrease ( $\mathrm{p}=0.0310)$. Takazawa et al. [4] demonstrated that the APG-d/a is a valuable index of left ventricular afterload, the evaluation of vasoactive agents, and the increasing 
APG-d/APG-a ratio represented a reduction of arterial stiffness. The decrease in SDPP-d/a could be related to a reduction in $\mathrm{LVdP} / \mathrm{dt}(\max )$ observed in a previous study [16] and probably with negative inotropic effect and decreased action of adrenergic activity.

The APG-d/APG-a ratio represented the blood flow in the aorta after the aortic valve opened, and the presence of blood flow in the aorta justifies the correlation between the APG-d/APG-a ratio and heart rate [19].

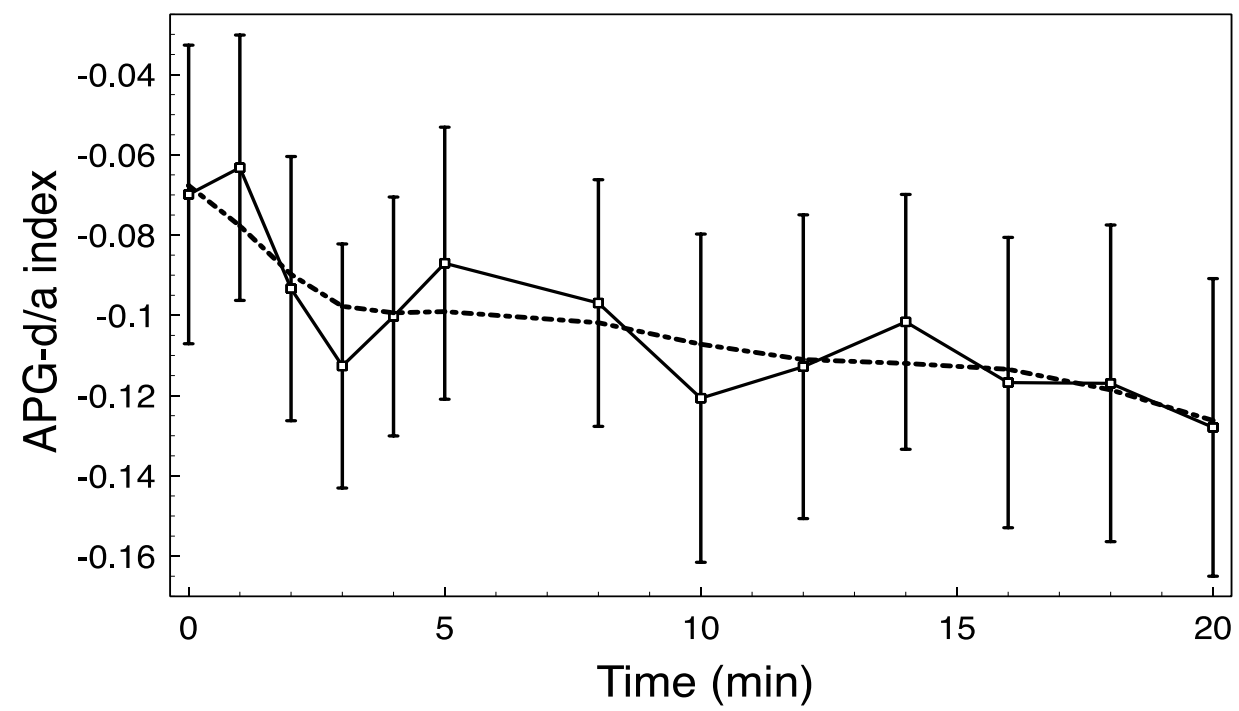

Fig.4. The second derivative of photoplethysmogram or acceleration photoplethysmogram d/a (APG-d/a) index with standard error. Smoothed template using the fast Fourier transformation (dotted line).

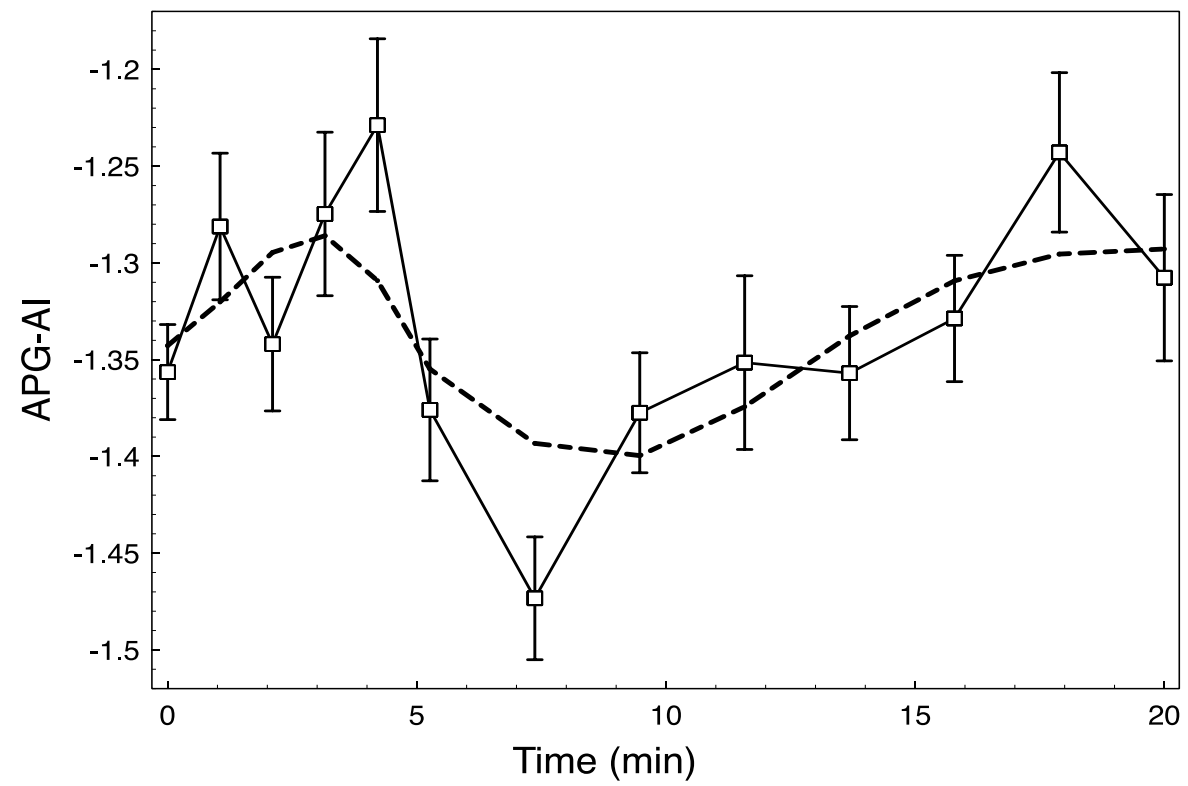

Fig.5. The second derivative of photoplethysmogram o acceleration photoplethysmogram aging index (APG-AI) with standard error. Smoothed template using the fast Fourier transformation (dotted line).

APG-AI. The changes in the APG-AI and the smoothed template using the fast Fourier transformation during and after manual acupuncture in PC6 are depicted in Figure 5. The acupuncture treatment elicited an APG-AI increase, and after needle withdrawal, a diminution; both changes were no significant. These changes can be related to modifications of mean arterial pressure, as has been reported in previous studies [16]. Takazawa et al. [4] found that 
the APG-AI increases with age and could be valuable for evaluating vascular aging and the screening of arteriosclerotic disease. Our experiments showed in the smoothing of APG-AI that the acupuncture elicited an acute biphasic behaviour with an increase in the acupuncture period and a secondary decrease response (Figure 5).

\section{Conclusions}

This study shows that the APG indices used as indicators of the general state of health and for clinical evaluation are acutely modified with the application of manual acupuncture in PC6. Besides, the FFT-smoothed spectrum of APG indices can be taken as a kinetic modeling to study the time-sequence of the effects of acupuncture on cardiovascular variables.

\section{Declarations}

\section{Source of Funding}

This research did not receive any specific grant from funding agencies in the public, commercial, or not-for-profit sectors.

\section{Competing Interests Statement}

The authors declare no competing financial, professional and personal interests.

\section{Consent to participate}

Not Applicable

\section{Consent for publication}

We declare that we consented for the publication of this research work.

\section{Availability of data and material}

Authors are willing to share data and material according to the relevant needs.

\section{Author's contribution}

All authors participated in overseeing laboratory work, data analysis, and manuscript writing and review. The corresponding author designed and supervised work.

\section{References}

1. Allen J (2007) Photoplethysmography and its applications in clinical physiological measurement. Physiol Meas 28:R1-R39.

2. Fleischhauer V, Ruprecht N, Sorelli M, Bocchi L, Zaunseder S. Pulse decomposition analysis in photoplethysmography imaging. Physiol Meas. 2020;41(9):095009.

3. Elgendi M. Standard terminologies for photoplethysmogram signals. Curr Cardiol Rev. 2012;8(3):215-9.

4. Takazawa K, Tanaka N, Fujita M, Matsuoka O, Saiki T, Aikawa M, Tamura S, Ibukiyama C. Assessment of vasoactive agents and vascular aging by the second derivative of photoplethysmogram waveform. Hypertension. 1998;32(2):365-70. 
5. Imanaga I, Hara H, Koyanagi S, Tanaka K. Correlation between wave components of the second derivative of plethysmogram and arterial distensibility. Jpn Heart J. 1998;39(6):775-84.

6. von Wowern E, Saldeen P, Olofsson P. Arterial stiffness during controlled ovarian hyperstimulation and early pregnancy in women exposed to assisted reproduction. Hypertens Pregnancy. 2018;37(4):182-91.

7. de Lima Pimentel R, Duque AP, Moreira BR, Rodrigues LF Junior. Acupuncture for the Treatment of Cardiovascular Diseases: A Systematic Review. J Acupunct Meridian Stud. 2019;12(2):43-51.

8. Vilaval T, Sasinan W, Mayuree C, Chananun P, Somchai S. Effect of acupuncture on blood pressure control in hypertensive patients. J Tradit Chin Med. 2019;39(2):246-250.

9. Shi L, Fang J, Zhao J, Liu G, Zhao Q, Zhang J, Zhang J, Zhu B, Liang F, Rong P. Comparison of the Therapeutic Effects of Acupuncture at PC6 and ST36 for Chronic Myocardial Ischemia. Evid Based Complement Alternat Med. 2017;2017:7358059.

10. Middlekauff HR. Acupuncture in the treatment of heart failure. Cardiol Rev. 2004;12(3):171-3.

11. Wang S, Ren L, Jia L, Gu Z, Fan X, Chen W, Hou P, Liang F, Rong P, Ma T, Liu X, Chen Y. Effect of acupuncture at Neiguan (PC 6) on cardiac function using echocardiography in myocardial ischemia rats induced by isoproterenol. J Tradit Chin Med. 2015;35(6):653-8.

12. Syuu Y, Matsubara H, Kiyooka T, Hosogi S, Mohri S, Araki J, Ohe T, Suga H. Cardiovascular beneficial effects of electroacupuncture at Neiguan (PC-6) acupoint in anesthetized open-chest dog. Jpn J Physiol. 2001;51(2):231-8.

13. Kim MS, Kim HD, Seo HD, Sawada K, Ishida M. The effect of acupuncture at PC-6 on the electroencephalogram and electrocardiogram. Am J Chin Med. 2008;36(3):481-91.

14. Li J, Li J, Chen Z, Liang F, Wu S, Wang H. The influence of PC6 on cardiovascular disorders: a review of central neural mechanisms. Acupunct Med. 2012;30(1):47-50.

15. Rivas-Vilchis JF, Hernández-Sánchez F, González-Camarena R, Suárez-Rodríguez LD, Escorcia-Gaona R, Cervantes-Reyes JA, Román-Ramos R. Assessment of the vascular effects of PC6 (Neiguan) using the second derivative of the finger photoplethysmogram in healthy and hypertensive subjects. Am $\mathrm{J}$ Chin Med. 2007;35(3):427-36.

16. Rivas-Vilchis JF, Gámez-Valdés E, Castañeda-Ramírez MS. Cardiovascular immediate response to PC6 manual acupuncture. Int J Sc Tech 2020;5(12):2217-20.

17. Heideman M, Johnson D, Burrus D. Gauss and the history of the fast Fourier transform. IEEE ASSP Magazine 1984;1(4):14-21.

18. Savitzky A, Golay MJE. Smoothing and differentiation of data by simplified least squares procedures. Ann Chem 1964;36:1627-39.

19. López-Beltrán EA, Blackshear PL, Finkelstein SM, Cohn JN. Non-invasive studies of peripheral vascular compliance using a non-occluding photoplethysmographic method. Med Biol Eng Comput. 1998;36(6):748-53. 